\title{
From primary instabilities to secondary instabilities in Görtler vortex flows
}

\author{
Xi Chen ${ }^{1,2}$, Jianqiang Chen ${ }^{1,2^{*}}$ (D), Xianxu Yuan ${ }^{1,2}$, Guohua Tu ${ }^{1,2}$ and Yifeng Zhang ${ }^{1,2}$
}

\author{
*Correspondence: jq-chen@263.net \\ ${ }^{1}$ State Key Laboratory of \\ Aerodynamics, China Aerodynamics \\ Research and Development Center, \\ Mianyang, China \\ ${ }^{2}$ Computational Aerodynamics \\ Institute, China Aerodynamics \\ Research and Development Center, \\ Mianyang, China
}

\begin{abstract}
We have studied the transformation process from primary instabilities to secondary instabilities with direct numerical simulations and stability theories (Spatial Biglobal and plane-marching parabolized stability equations) in detail. First Mack mode and second Mack mode are shown to be able to evolve into the sinuous mode and the varicose mode of secondary instability, respectively. Although the characteristics of second Mack mode eventually lose in the downstream due to the synchronization with the continuous spectrum, second Mack mode is found to be able to trigger a sequence of mode resonations which in turn give birth to highly unstable secondary instabilities. In contrast, first Mack mode does not involve in any mode synchronization. Nevertheless, it can still "jump" to a sinuous mode of secondary instability with a much larger growth rate than that of the first Mack mode. Therefore, secondary instabilities of Görtler vortices are highly receptive to the primary instabilities in the upstream, so that one should consider the primary instability in the upstream and the secondary instability in the downstream as a whole in order to get an accurate prediction of the boundary layer transition.
\end{abstract}

Keywords: Primary instability, Secondary instability, Görtler vortices

\section{Introduction}

Görtler instability, manifesting itself as counter-rotating streamwise vortices, is frequently encountered in boundary layer flows over concave walls and other near-wall flows having curved streamlines. After extensive studies on this subject, especially in incompressible flows, the linear evolution of Görtler vortices have become well known [1-3] while receptivity and breakdown mechanisms are far from being completely understood. Görtler vortices can be excited by freestream disturbances [4-6] or wall inhomogeneities (e.g., roughness, blowing and suction)[7-9]. With controlled inlet forcing, investigators usually obtained a clean flow where Görtler vortices possess only a single dominant wavelength, and almost all the stability analyses have been performed for such flows [10, 11]. In some cases, however, vortex generators can trigger the first harmonic with a comparable amplitude with the primary vortex. If the harmonic turns out to be linearly unstable, then the harmonic can grow and form secondary streaks between the primary streaks induced by the primary vortex. Secondary streaks have been reported in both experimental and numerical studies [9, 12-15], but the corresponding stability characteristics remain unknown until quite recently. [16] have utilized blowing and suction to numerically excite Görtler vortices in a Mach 6.5 concave boundary layer. They found

(c) The Author(s). 2019 Open Access This article is distributed under the terms of the Creative Commons Attribution 4.0 International License (http://creativecommons.org/licenses/by/4.0/), which permits unrestricted use, distribution, and reproduction in any medium, provided you give appropriate credit to the original author(s) and the source, provide a link to the Creative Commons license, and indicate if changes were made. 
that large-wavelength blowing and suction tends to trigger secondary streaks in addition to primary streaks. With DNS and detailed stability analyses, they have found that the boundary layers with secondary streaks are subject to more kinds of secondary-instability modes with generally larger growth rates, and hence easier to transition.

At the initial stage when Görtler vortices are still small, the boundary layer is governed by primary instabilities which are somewhat modulated by Görtler vortices. Farther downstream as the Görtler vortices develop mushroom structures, secondary instability would be dominant. Two distinguished types of secondary-instability modes, i.e., varicose and sinuous modes, are frequently encountered [17], and are thus of most interest. The varicose mode possesses a symmetrical streamwise velocity distribution while the sinuous mode has a antisymmetrical one. The sinuous mode was usually believed to dominate the transition in hypersonic boundary layers $[11,18]$. However, the varicose mode might be more dangerous if considering the presence of secondary streaks and the possibilities of the transformation of primary instabilities to secondary instabilities as revealed by [16].

Primary-secondary instability transformation, or the receptivity process of secondary instabilities to the primary instabilities, is not only present on the concave wall. Rather, it appears to be a common process during the boundary layer transition. Previous studies [19-21] indicate that the primary-secondary instability transfer could have at least two routes. The first route is through continuous modulation where the mode identity remains unchanged in the sense of local stability analysis during the transformation. In this case, the secondary-instability mode can be traced back to one of the unstable modes of the discrete spectrum for the otherwise laminar boundary layer in the upstream. The subharmonic sinuous mode originating from the first Mack mode in the supersonic streak flow identified by $[19,20]$ corresponds to this type. For the second route, the secondaryinstability mode originates from one of the continuous spectra of the otherwise laminar boundary layer, but at the same time has a part of frequency region merging with the frequency region of the primary instability in the upstream. Then due to the nonparallel effect, the primary-instability mode might "jump" to the secondary-instability mode with a much larger growth rate than that of the primary one. The transformation of Görtler vortices to the secondary-instability mode of the second Mack mode in a Mach 6 flaredcone boundary layer studied by [21] belongs to this case. In this paper, we find another route where the primary instability could translate to the secondary instability through mode synchronization.

Although [16] have indicated that the primary instabilities can turn into secondary instabilities in Görtler vortex flow, the details of the transformation are unclear yet, since they have only performed local stability analysis at a few of streamwise locations. In this paper, extensive local stability analyses based on a spatial approach are performed for a much larger number of streamwise locations. Plane-marching parabolized stability equations (PSE3D), are also utilized to trace the downstream evolution of the fixedfrequency disturbances. Moreover, DNS with a controlled forcing are also performed to verify the results of stability theory.

\section{Numerical settings and linear stability theory}

\subsection{Direct numerical simulations}

In this subsection we briefly introduce the model and numerical settings of DNS. The model consists of a sharp flat plate and a concave afterbody generated by a circular arc, 
as sketched in Fig. 1. The inlet of the simulation is at $x=10 \mathrm{~mm}$ from the leading edge, where a similarity solution is forced. The leading-edge shock is weak (since the edge is sharp) and far away from the boundary layer in the downstream, hence is not considered here. No-slip and adiabatic conditions are prescribed at the wall. The wall-normal velocity component at the wall is specified in the primary blowing-suction strip region as follows:

$$
\begin{array}{r}
v_{b s}(x, 0, z, t)=\mathcal{A}_{0} \sin ^{3}\left(\pi\left(x-x_{1}\right) /\left(x-x_{2}\right)\right) \cos (\beta z), \quad x_{1} \leq x \leq x_{2}, \\
x_{1}=56.5 \mathrm{~mm}, x_{2}=62.6 \mathrm{~mm},
\end{array}
$$

where $\mathcal{A}_{0}$ is set to be $15 \%$ of the freestream velocity, $\beta$ the spanwise wavenumber of the blowing and suction. Note that large-amplitude blowing and suction is used in order to obtain an earlier saturation state of Görtler vortices. In this paper a single blowing-suction spanwise wavelength, i.e., $6 \mathrm{~mm}$, is considered. High-frequency disturbances are forced in the secondary blowing-suction region located between $75 \mathrm{~mm}$ and $78 \mathrm{~mm}$ if needed.

The software OPENCFD, developed by [22], was used for the DNS. Viscous terms are discretized with an eighth-order centred finite-difference scheme. Convection terms are discretized with a seventh-order weighted essentially non-oscillatory (WENO) scheme for transition simulation and with a seventh-order upwind scheme for laminar simulation where Görtler vortices do not break down. The transitional simulation is focused on the breakdown process of Görtler vortices, whereas the laminar simulation is performed to provide the base flow for stability analyses and validation of stability theory (see the Appendix A). The detailed simulation setting can be found in Table 1. A third-order total variation diminishing-type Runge-Kutta method is used for the time stepping. The physical domain is resolved by $1000 \times 201 \times 150$ grid points in the streamwise, wall-normal and spanwise directions, respectively, giving a total of 30 million points. The grid resolution is found to be sufficient for subsequent stability analyses.

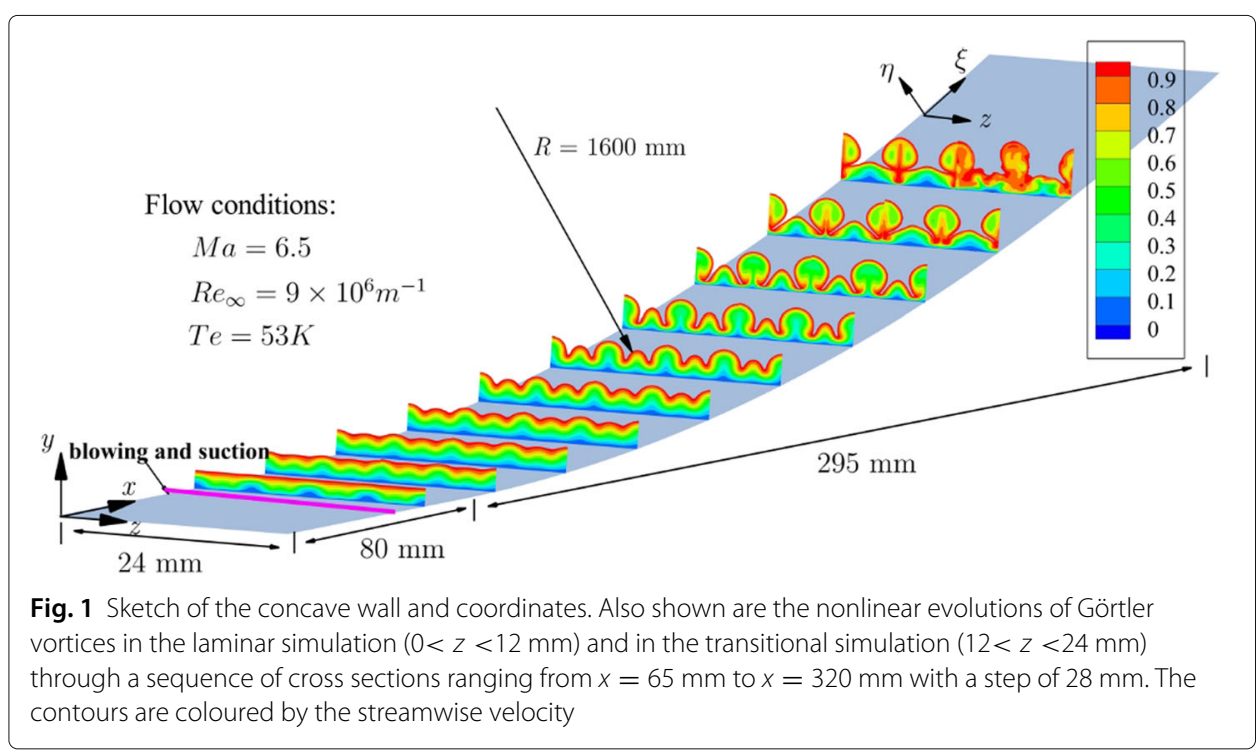


Table 1 Certain numerical settings for the transitional simulation and the laminar simulation

\begin{tabular}{llll}
\hline cases & scheme & primary & secondary \\
& for convection terms & blowing-suction strip & blowing-suction strip \\
\hline Transitional simulation & 7th WENO & $15 \% U_{e}$, steady & $2 \%$, random \\
Laminar simulation & 7th upwind & $15 \% U_{e}$, steady & $0.002 \%$, single frequency \\
\hline
\end{tabular}

\subsection{Local stability analysis: BiGlobal method}

We consider the stability characteristics in a cross-section by decomposing the flow field as follows

$$
q(\xi, \eta, z, t)=\bar{q}(\eta, z)+\hat{q}(\eta, z) \exp (i \alpha \xi-i \omega t)+c . c .,
$$

where $q=(u, v, p, T, w), \bar{q}$ the basic states, $\hat{q}$ the shape function of the disturbances. $\alpha$ represents the streamwise wavenumber. $\omega$ is the angular frequency with the corresponding dimensional frequency denoted by $f$. After substituting the above decompositions into the Navier-Stokes equations, subtracting the basic states and neglecting the non-parallel and nonlinear terms, one obtains the eigenvalue problems as

$$
\left(\begin{array}{cc}
0 & 1 \\
-\mathcal{A}(\eta, z) & -\mathcal{B}(\eta, z)
\end{array}\right)\left(\begin{array}{c}
\hat{q} \\
\alpha \hat{q}
\end{array}\right)=\alpha\left(\begin{array}{cc}
1 & 0 \\
0 & \mathcal{C}(\eta, z)
\end{array}\right)\left(\begin{array}{c}
\hat{q} \\
\alpha \hat{q}
\end{array}\right)
$$

for the spatial approach where $\alpha$ is to be solved with $\omega$ given. The growth rate is $-\alpha_{i}$ (the imaginary part of $\alpha$ ). Dirichlet boundary conditions are forced for all the variables at wall and infinity, except for the temperature disturbances at wall where Neumann boundary condition is used in consistence of DNS. $\mathcal{A}, \mathcal{B}$ and $\mathcal{C}$ are linear operators, of which the wall-normal differentials are discretized using the fourth order finite difference scheme. Thanks to the symmetry of the base flow and eigenfunctions, we only need to consider half of the spanwise wavelength, and apply Fourier cosine and sine collocation schemes [23] for symmetrical and antisymmetrical eigenfunctions, respectively. The eigenvalues are then determined by using Arnoldi's method.

\subsection{Marching stability analysis: PSE3D method}

In contrast to the local stability analysis introduced above, PSE3D incorporate initial conditions and nonparallel effects. In the PSE3D formulation, the disturbance is decomposed into a rapidly varying wave-like part and a slowly varying shape function as follows

$$
q(\xi, \eta, z, t)=\bar{q}(\xi, \eta, z)+\hat{q}(\xi, \eta, z) \exp \left(i \int_{\xi} \alpha \mathrm{d} \xi-i \omega t\right)+\text { c.c. }
$$

where $\hat{q}(\xi, \eta, z)$ is assumed to vary slowly with $\xi$ so that $\partial^{2} \hat{q} / \partial \xi^{2} \ll 1$. Substituting (4) into Navier-Stokes equations, and negecting nonlinear terms as well as higher derivatives of $\hat{q}$ with respect to $x$, yields linear PSE3D equations as

$$
\mathcal{L} \hat{q}+\mathcal{M} \frac{\partial \hat{q}}{\partial \xi}=0 .
$$

Where $\mathcal{L}$ and $\mathcal{M}$ are linear operators discretized by the same schemes as used in BiGlobal. Equations (5) are solved using backward Euler method with the initial profiles provided by BiGlobal. To avoid the ambiguity in the $\xi$-dependence between $\hat{q}$ and $\alpha$, the wavenumber at each station was updated as

$$
\alpha^{\text {new }}=\alpha^{\text {old }}-i \frac{1}{E} \int_{\eta} \int_{z} \bar{\rho}\left(\hat{u}^{*} \frac{\partial \hat{u}}{\partial \xi}+\hat{v}^{*} \frac{\partial \hat{v}}{\partial \xi}+\hat{w}^{*} \frac{\partial \hat{w}}{\partial \xi}\right) d \eta d z,
$$


where

$$
E=\int_{z} \int_{\eta} \bar{\rho}\left(|\hat{u}|^{2}+|\hat{v}|^{2}+|\hat{w}|^{2}\right) d \eta d z
$$

and the asterisk denotes the complex conjugate. This iteration continued until the latest change was less than $10^{-5}$. Note that the streamwise curvature has been included in the linear operators of both BiGlobal and PSE3D.

\section{Results and analysis}

Nonlinear evolutions of Görtler vortices in the laminar simulation and the transition simulation are both shown in Fig. 1. It can be seen that the wave profiles initiated by blowing and suction evolve into mushroom structures in the downstream. In addition to the primary streaks developed right downstream of the blowing region $\left(v_{b s}>0\right.$, see the function 1), secondary streaks also emerge downstream of the suction region $\left(v_{b s}<0\right)$. According to the previous study by [16], the Görtler vortices would reach saturated at around $250 \mathrm{~mm}$. Further downstream, the mushroom structures persist in the laminar simulation while break down in the transitional simulation, as illustrated in Fig. 1.

\subsection{From the primary instability to the varicose mode of the secondary instability}

At first, we examine how the primary instabilities evolve in the Görtler vortex flow with PSE3D. The base flow is chosen to be the time-averaged flow field of the transitional simulation so that the PSE3D results can be compared with the DNS results. Figure 2(a) shows the streamwise variations of growth rates of varicose modes with various frequencies. For simplicity, we denote the varicose modes originated from the first-mode instability and the second-mode instability as first-varicose modes and second-varicose modes, respectively. It is found that the second-varicose modes are dominant with the peak frequency of 135 $\mathrm{kHz}$ (the red line), while the most amplified component of the first-varicose modes is of
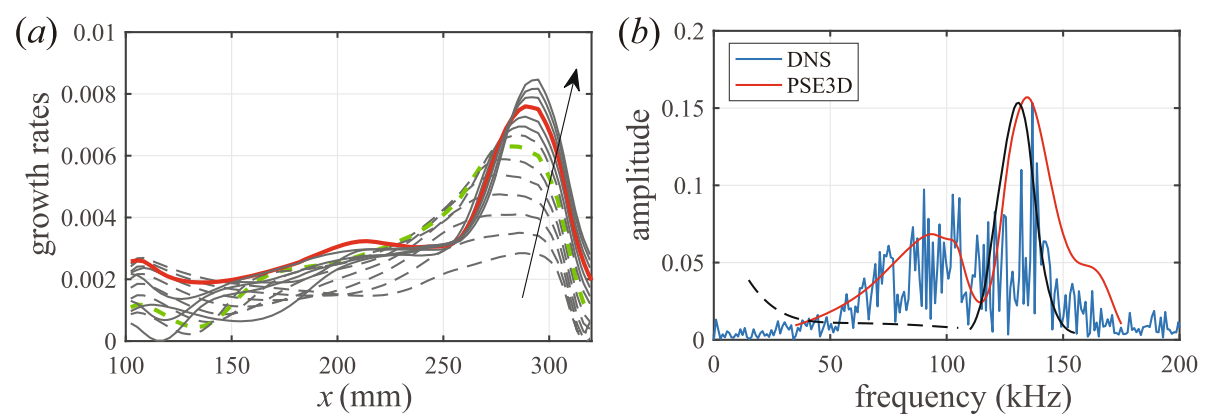

Fig. 2 (a) Evolution of growth rates of varicose modes obtained by PSE3D for various frequencies ranged from $35 \mathrm{kHz}$ to $165 \mathrm{kHz}$ with step of $10 \mathrm{kHz}$. The arrow indicates the increasing direction of frequency. The solid lines represent the second-varicose modes, while the dashed lines represent the first-varicose modes. The thick lines denote the largest components of the first- and second-varicose modes. (b) Comparison of spectra obtained by DNS (the transitional simulation) and PSE3D at $x=289 \mathrm{~mm}$. The spectrum of DNS is obtained by Fourier analysis of the time series at the largest root-mean-square (rms) point in the center line of the primary streak, which is believed to be contributed by only varicose modes. The peak value of the PSE3D results is fixed to be equal to that of the DNS results. The spectrum obtained by the classical PSE (not accounting for the spanwise variations of the mean flow) for the laminar boundary layer (i.e., in absence of Görtler vortices) is also shown (black lines). The solid black line and dashed black line stand for the planar and oblique results (with the spanwise wavelength of $6 \mathrm{~mm}$ ), respectively. Note that the dashed-line results have been magnified by ten 
$95 \mathrm{kHz}$ (the green line). After being at a moderate level for a long term, the growth rates of second-varicose modes experience a rapid increase when the Görtler vortices become saturated $(250 \mathrm{~mm})$. The rapid growth of the amplification rates indicates the appearance of secondary instability. It is interesting to note that for second-varicose modes the primary-instability growth rates (i.e., the growth rates before $250 \mathrm{~mm}$ ) are more sensitive to frequency than the secondary-instability growth rates. This observation indicates that the second-mode instability in the upstream, rather than the secondary instability in the downstream, determines the final spectrum of the second-varicose modes. This statement is further supported by the fact that the spectrum at $x=289 \mathrm{~mm}$ for the laminar boundary layer (black line) is close to that for the Görtler vortex flow, as shown in Fig. 2(b). The latter shifts to a slightly higher frequency region due to the secondaryinstability effects. In contrast, the growth rates of first-varicose modes vary remarkably with frequency, implying that the secondary instability plays an important role in shaping the spectrum of the first-varicose modes. This explains why the first-varicose spectrum for the Görtler vortex flow is completely different with that for the laminar case (dashed black line in Fig. 2(b)). The spectrum predicted by PSE3D is further compared with that obtained by DNS. The agreement is excellent, considering that PSE3D has neglected the receptivity process by assuming the same initial amplitude for each component.

In order to further understand the transformation process of primary-secondary instability, we consider the frequency component, $135 \mathrm{kHz}$, in detail, with spatial BiGlobal method. The mode traces are shown in Fig. 3. The results are based on the laminar simulation, hence the Görtler vortices do not break down and the secondary-instability growth rates do not rapidly decrease at last as in the transition simulation. It can be seen that multiple unstable modes will emerge in the downstream and they are denoted as "V1, V2, ...," according to the order in which they appear. In particular, mode V1 is found to originate from the planar second-mode instability, while others can be traced back to continuous spectra.

The most interesting feature is that mode V1 would trigger a series of mode resonations at synchronization regions (denoted as R1 through R5) where two modes have close phase velocities. As suggested by [24], exact synchronization point (usually a complex Reynolds number) can be determined by solving the equation below

$$
\left\langle\mathcal{H} \phi, \phi_{a}\right\rangle \equiv \int_{\eta} \int_{z} \phi \mathcal{H} \phi_{a}^{+} \mathrm{d} \eta \mathrm{d} z=0,
$$

where $\phi \equiv(\hat{q}, \alpha \hat{q}), \phi_{a}$ and $\mathcal{H}$ are the eigenfunction, the eigenfunction of the adjoint problem and the linear operator of the right hand of Eq. (3), respectively. The upscript ${ }^{+}$ denotes the conjugate part. For real Reynolds numbers or real streamwise locations as in our case, it is convenient to define " $S$ factor" as follows to indicate the synchronization degree,

$$
S \equiv \frac{\left\langle\mathcal{H} \phi, \phi_{a}\right\rangle}{\sqrt{\langle\phi, \phi\rangle} \sqrt{\left\langle\phi_{a}, \phi_{a}\right\rangle}} .
$$

Smaller $S$ means higher degree of synchronization, or in other words that the mode is closer to synchronization. Evolution of $S$ factors for modes V1, V2, V3 and V6 are shown in Fig. 3(c). It can be seen that the synchronization regions as labeled in Fig. 3(a,b) coincide with the valleys of $S$ factors. In particular, when two modes are synchronized, their 

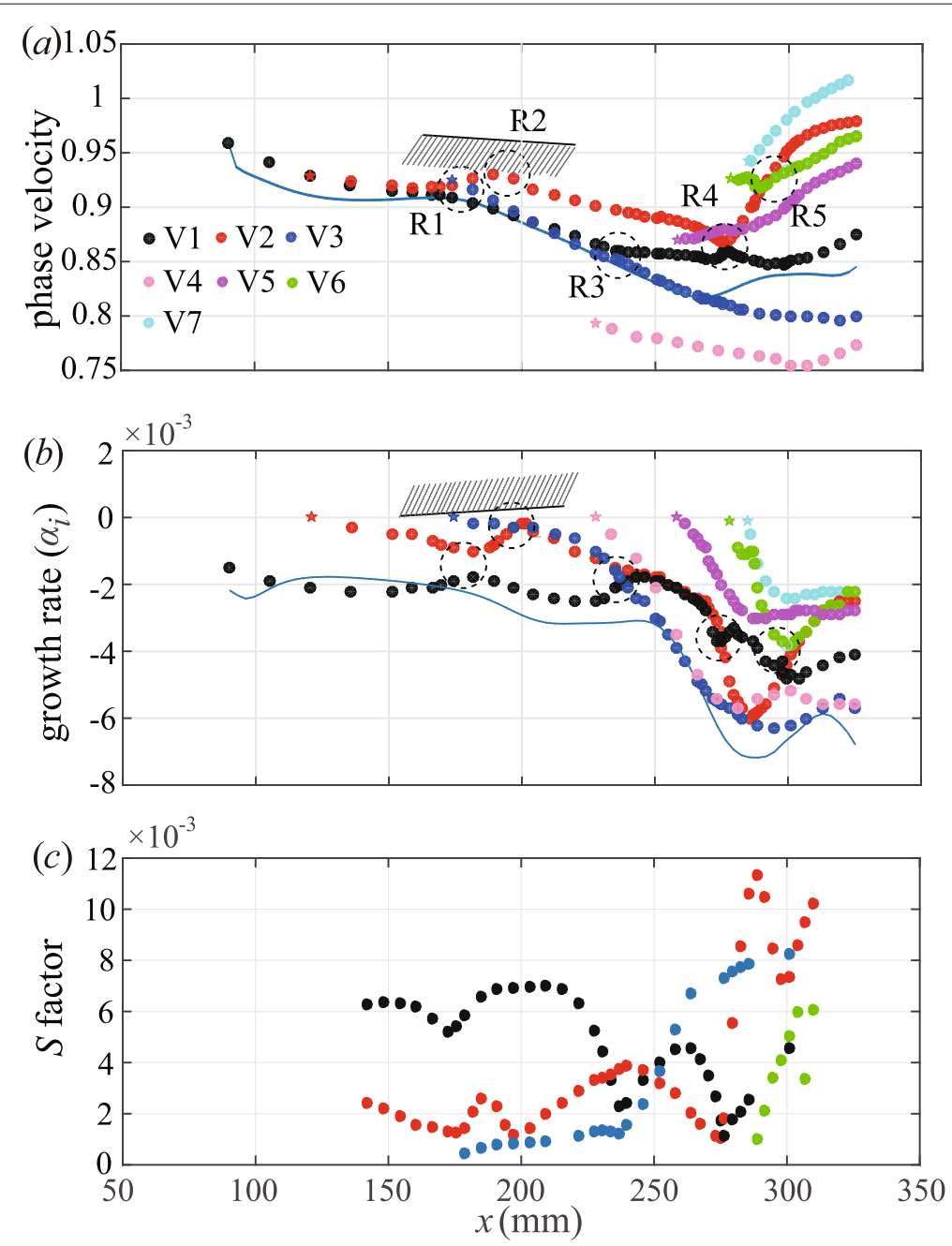

Fig. 3 BiGlobal results showing traces of different varicose modes with frequency of $135 \mathrm{kHz}$, (a) phase velocities, (b) growth rates and (c) $S$ factor. The star, $\star$, depicts the neutral location where the mode becomes unstable. The solid line represents the PSE3D results initiated by mode V1. Mode synchronization regions are indicated by dashed circles. The black line denotes the continuous spectrum and the grey region depicts the nearby stable modes

S factors both nearly reach a minimum. This observation is consistent with the definition of $S$ factor which plays as an indicator of synchronization.

Mode resonations might lead to rapid changes of phase velocities, growth rates as well as mode shapes (see also Fig. 4). For region R1, the evolution trends of growth rates of modes V1 and V2 change to the opposite during the mode synchronization. Meanwhile, mode $\mathrm{V} 2$ gradually possesses a notable distribution below the relative sonic line, a typical characteristic of the second-mode instability which mode V1 eventually loses in contrast, as shown in Fig. 4(a,b). In that sense, synchronization in R1 seems to lead to a mode exchange. For the sake of continuous evolution, we still refer to the black symbols and red symbols as mode V1 and mode V2, respectively. Further downstream, mode V2 meets a continuous spectrum (which is likely the vortex/entropy spectrum modulated by Görtler vortices) and soon departs with the second-mode characteristics lost. On the other hand, mode V1 successively synchronizes with the secondary-streak mode V3 (whose mode 


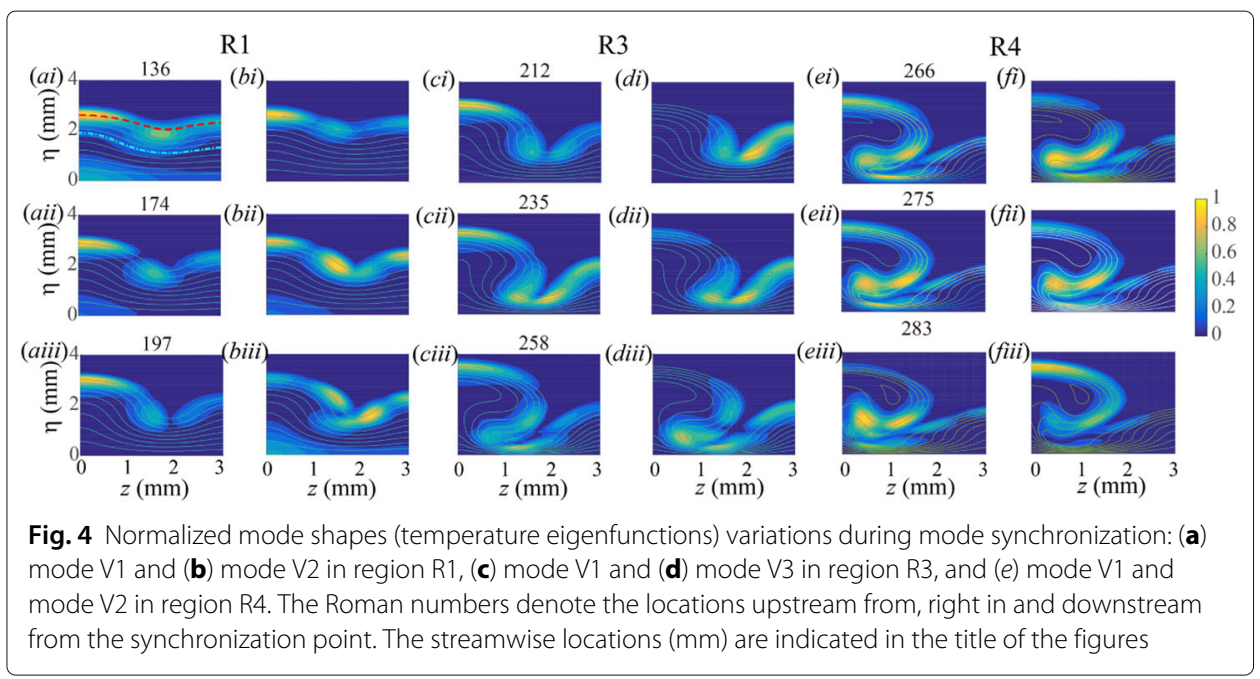

shape mainly locates at the secondary streak) in region R3 and again with mode V2 in region R4. Synchronization R3 notably enhances the instability of mode V3 while reduces the growth rate of mode V1. Downstream from region R3, secondary instabilities manifest themselves as indicated in PSE3D results. Mode resonation in R4 stabilizes mode V1, but promotes mode V2. The abrupt changes of phase velocities and mode shapes (shown in Fig. $4(e, f))$ indicate that synchronization R4 also causes a mode exchange like R1. In contrast, the mode shapes of modes V1 and V3 (Fig. 4(c,d)) remain essentially the same after crossing region R3, indicating no mode exchange occurs there.

At last, modes V2 and V6 are in synchronization in region R5. Apart from perturbations on the phase velocities and growth rates (especially for mode V6), synchronization results in notable changes of eigenfunctions for both modes. As shown in Fig. 5( $a, b)$, mode V2 and mode V6 are both strengthened in the shoulder region of the primary mushroom structure after synchronization. The real parts of the mode shapes (Fig. 5(c,d)) further reveal that isolated lobes emerge and form a nearly closed loop. Such multiplelobe structures resemble the shape functions of amplified eigenmodes of the Batchelor vortex (i.e., trailing line vortices) (see, e.g., [25]). This similarity is not surprising, since the Görtler vortices, with being continuously lifting up due to the self-induction of the counter-rotating vortex rolls, would behave more like the free trailing line vortices in the downstream. The change of mode shape can be further illustrated when considering the streamwise periodicity of the mode, as shown in Fig. $5(e, f)$. It can be seen that after synchronization the upper and lower parts of the shape function of mode V2 are strongly stretched to form hairpin-like structures, while the backward inclined structures of mode V6 are up elongated to form a helical structure.

In deed, helical structures seem to be one of dominant structures in the fully developed Görtler vortices, as shown in Fig. 6. One can easily observe that the first four modes, i.e., modes V7, V2, V6 and V5, all exhibit helical structures. Since helical structures often emerge with the helical modes of instability identified in trailing vortices flows, these four modes might be classified as helical modes. The other three modes either concentrate in the inner part of the mushroom structure (modes V1 and V4) or in the top of the secondary streak (mode V3), and hence can be referred to as inner modes and secondarystreak mode, respectively. 


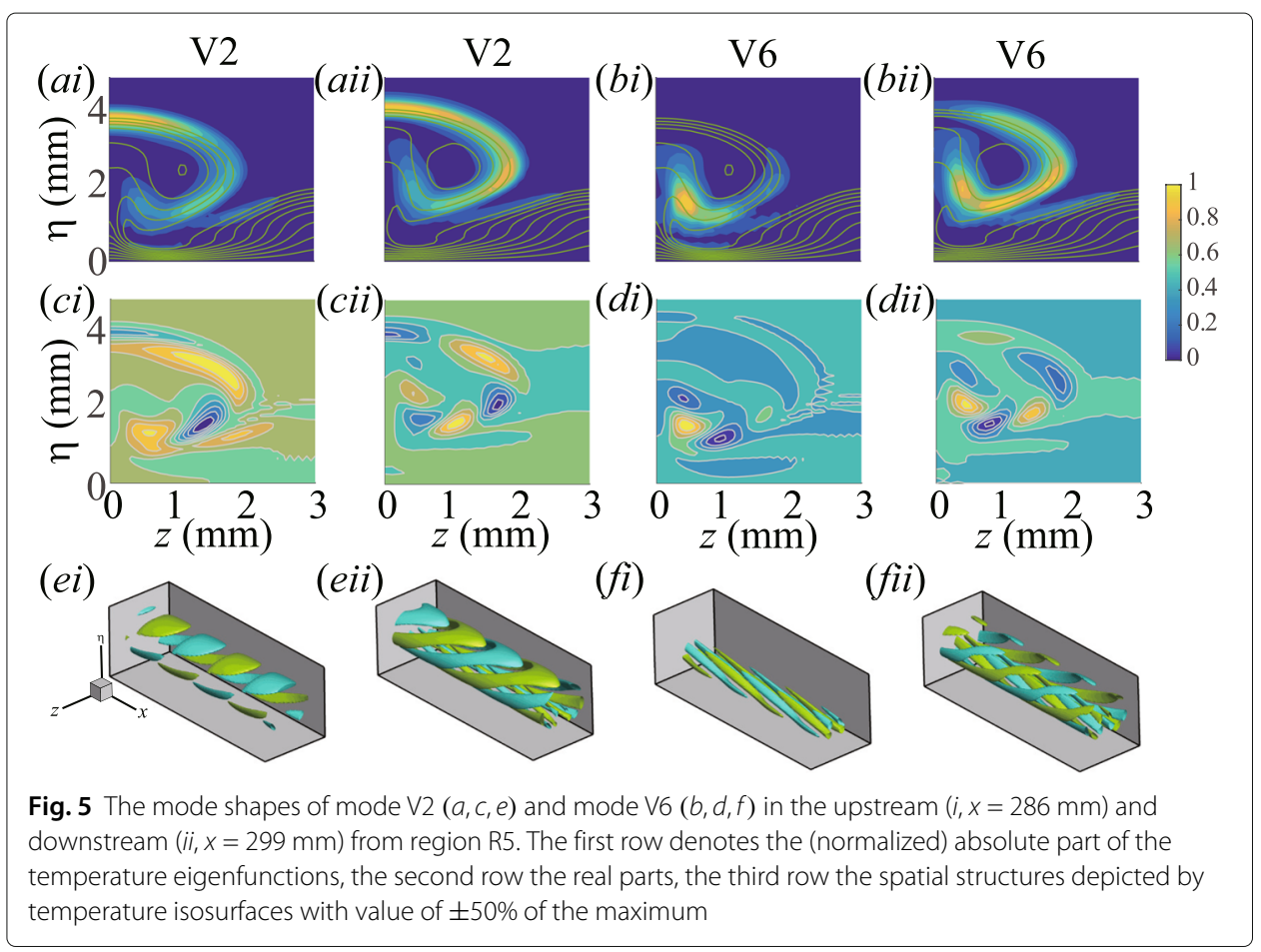

The PSE3D results initiated by mode V1 (or modulated second Mack mode) are also shown in Fig. 3. It can be seen that the modulated second Mack mode initially follows the evolution of mode V1, then takes the route of mode V3 after passing region R3, and departs at around $x=270 \mathrm{~mm}$ due to effects of other unstable modes. In other words, the disturbances in the upstream tend to take a most dangerous route so that they can get the largest amplification. It needs to be noted that the disturbances in the secondaryinstability stage should be viewed as a combination of several unstable modes rather than

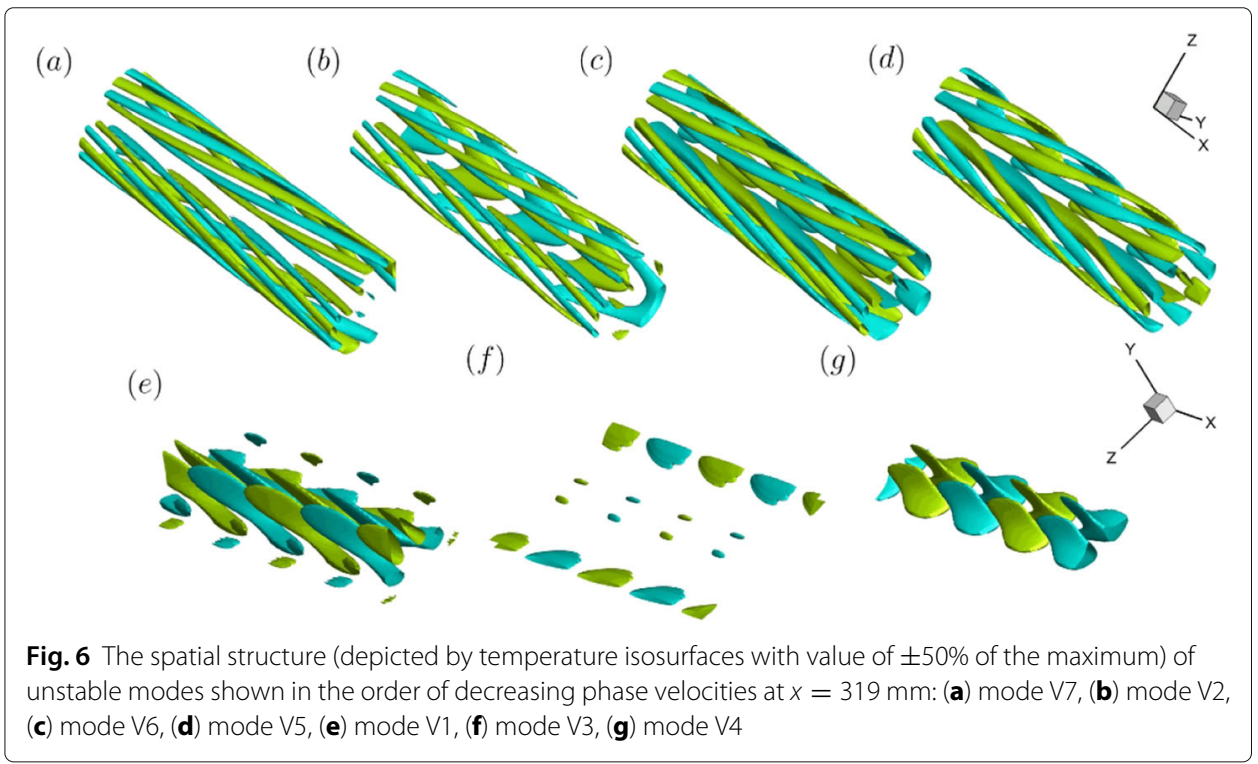


a single mode, since a few of unstable modes possess comparable growth rates in that stage.

The traces of the first-varicose mode of $95 \mathrm{kHz}$ have also been calculated (not shown here), and the results are qualitatively similar with those of the second-varicose mode. In particular, mode synchronization like R3 also occurs right before the saturation of Görtler vortices. However, it appears too weak to cause a remarkable promotion of instability, as is consistent with the growth rates evolution predicted by PSE3D shown in Fig. 2(b).

\subsection{From the first-mode instability to the sinuous mode of the secondary instability}

We further consider the transformation from the first Mack mode to the sinuous mode of secondary instability. The frequency is chosen to be equal to $95 \mathrm{kHz}$, the components of which are shown to be most amplified for the sinuous-mode instability by [16]. In contrast to the varicose-mode case above, only two significantly unstable modes, i.e., mode S1 and mode S2, exist in this case and no prominent mode synchronization is observed, as shown in Fig. 7. Mode S1 traces back to the first Mack mode, whereas mode S2 emerges at $x \approx 220 \mathrm{~mm}$ with lower phase velocities and higher growth rates than mode S1. PSE3D results show that the disturbances forced by mode $\mathrm{S} 1$ at inlet initially follow the route of mode S1, then quickly deviate towards the route of mode S2 when mode S2 appears, and roughly remain in that route after $x>270 \mathrm{~mm}$. Therefore, the disturbances also tend to take the route with largest amplification rates as in the above case.

\section{Discussion}

Indeed, mode synchronization also exists in laminar hypersonic boundary layers (in absence of Görtler vortices), and is shown to play a key role in the receptivity of primary instabilities [24, 26]. According to the theory of [24], the normal mode decomposition is not valid near the synchronization region and local method like the multiple-mode method should be used to reveal the details of modal interactions (nonparallel effects in nature). In our case, mode synchronization generally involves two two-dimensional unstable modes rather than two one-dimensional stable (or one stable and the other unstable) modes in the laminar case. Nevertheless, the local behaviors of mode synchronization in both cases are similar and are likely described by the same mechanism.
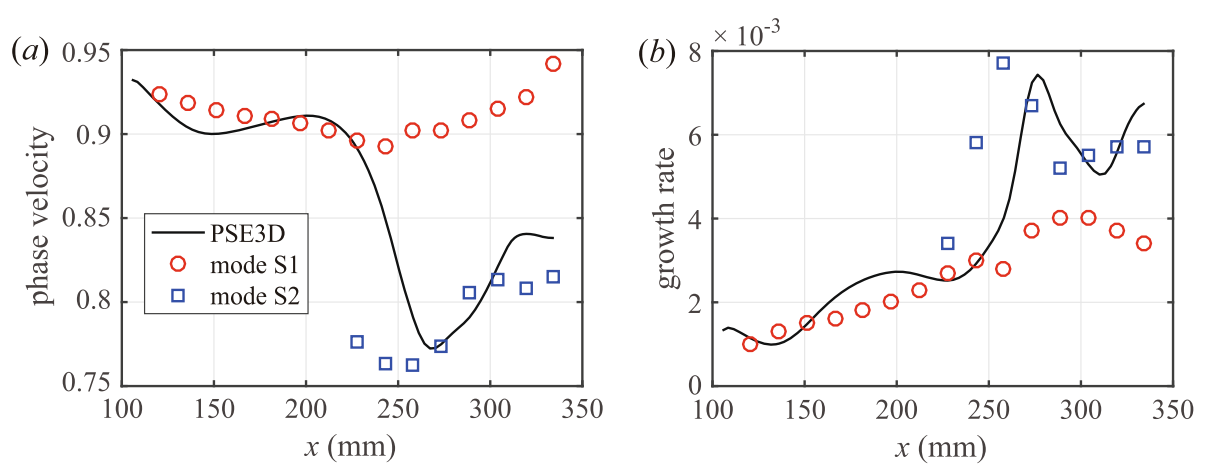

Fig. 7 PSE3D results showing the evolution of phase velocity $(\mathbf{a})$ and growth rate $(\mathbf{b})$ for the first-sinuous mode with frequency of $95 \mathrm{kHz}$. The BiGlobal results are indicated by the symbols 
Here, we only present results on Görtler vortices with secondary streaks. Although secondary streaks are frequently encountered in practically triggering Görtler vortices as introduced in the Introduction, whether such primary-secondary instability transformation exists in Görtler vortices without secondary streaks still requires further investigation. Here we could make several notes about this issue. Firstly, mode synchronization is sensitive to the changes of base flow as shown in [24]. Therefore, secondary streaks would surely play an important role in the primary-secondary instability transformation especially for the second-varicose case. In that case, mode V3 no longer exists if the secondary streaks are eliminated, and consequently synchronization R3 as well as the following ones might also disappear. On the other hand, synchronization R1 might not be qualitatively affected since it is between the planar and oblique components of second-mode instability. As a result, the planar second-mode components might still merge with a continuous spectrum, while the oblique second-mode components would be moderately enhanced like the case studied by [27] as it travels downstream.

\section{Conclusions}

In this paper, we have shown that the second Mack mode can trigger secondary instability through a sequence of mode synchronization, while the first Mack mode can "jump" to a new type of secondary-instability mode with a much larger growth rate. Our results confirm the speculation of [16] that the second Mack mode can "continuously" develop into the varicose mode of secondary instability while the first Mack mode would transfer to the sinuous mode of secondary instability through a phase velocity jump. As a byproduct, we also show that hairpin structures can develop from varicose modes through mode synchronization without sorting nonlinear effects for help.

\section{Appendix A}

\section{Cross validation of PSE3D and DNS results}

In the laminar simulation, two-dimensional blowing and suction with frequency of 135 $\mathrm{kHz}$ and three dimensional blowing and suction with frequency of $95 \mathrm{kHz}$ are utilized to excite the varicose mode and the sinuous mode in the downstream, respectively. The evolution of the mode amplitude, defined as the maximum amplitude of temperature
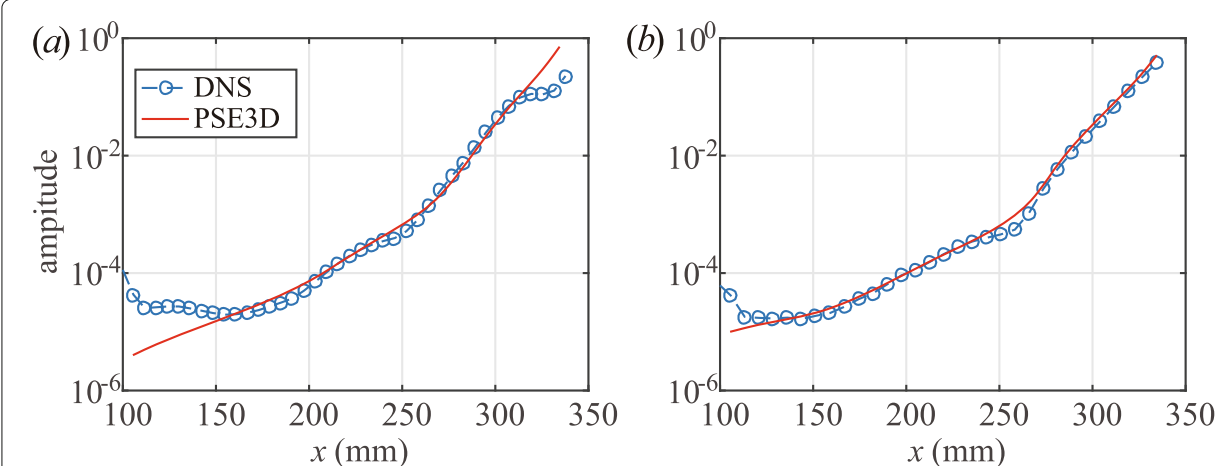

Fig. 8 Comparison of amplitude evolutions from DNS and PSE3D results for (a) the varicose mode of $135 \mathrm{kHz}$ and (b) the sinuous mode of $95 \mathrm{kHz}$. The amplitude is defined as the maximum of the temperature disturbances 


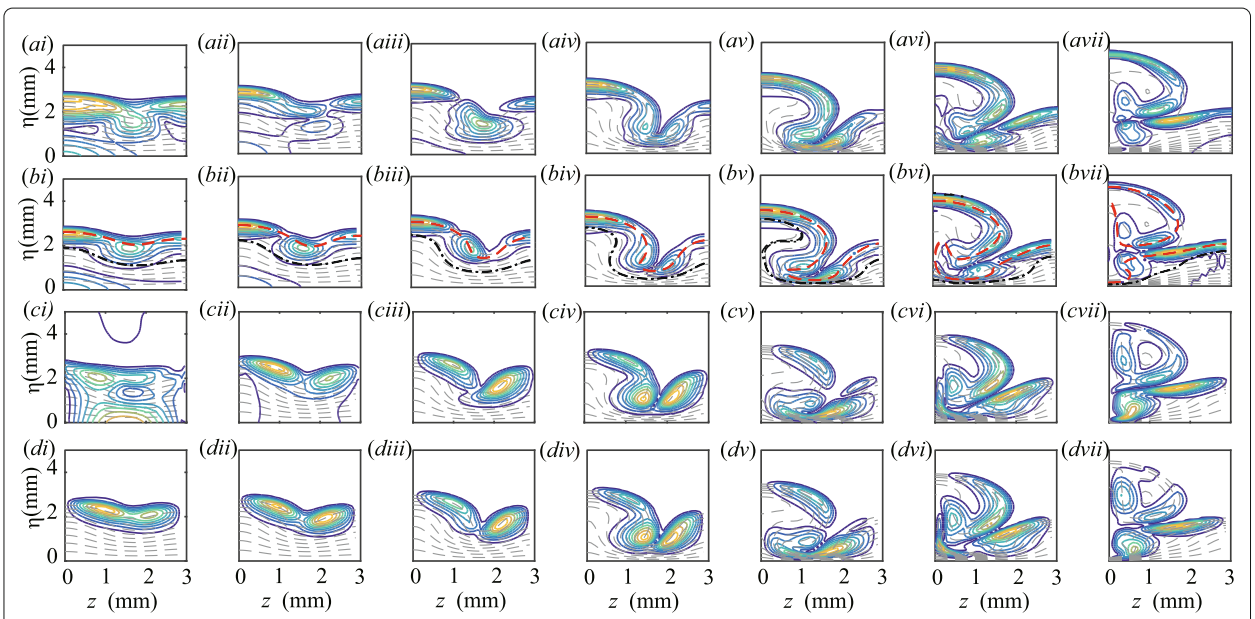

Fig. 9 Isolines of normalized temperature disturbances of the varicose mode of $135 \mathrm{kHz}(a, b)$ and the sinuous mode of $95 \mathrm{kHz}(c, d)$ at various streamwise locations obtained by DNS $(a, c)$ and PSE3D $(b, d)$, together with the base flow (dashed lines). Critical layer (dashed red) and relative sonic line (dash-doted black) are also plotted in the row (b). The Roman numbers denote the streamwise locations (i) $136 \mathrm{~mm}$, (ii) 167 mm, (iii) 197 mm, (iv) 228 mm, (v) 258 mm, (vi) 289 mm, (vii) 319 mm

disturbances, are shown in Fig. 8, together with the PSE3D results. For both cases, PSE3D results and DNS results are in excelent agreement.

The downstream evolutions of the mode shapes obtained from PSE3D and DNS results are shown in Fig. 9. Again, good agreement can be observed. The varicose mode shape initially exhibits a notable distribution below the relative sonic line, which is a prominent feature of the second Mack mode. Due to the increasing modulation of Görtler vortices, the near-wall components gradually disappear and the temperature disturbances concentrate in the vicinity of critical layer. This is different with the cross-flow case where second Mack mode seems to persist in its characteristic in the secondary-instability stage of the crossflow vortices as shown in [28].

\section{Abbreviations}

$x, y, z$ : Cartesian coordinates in the streamwise, wall-normal and spanwise directions, respectively; $\xi, \eta, z:$ Body-oriented coordinates, shown in Fig. $1 ; v_{b s}, \mathcal{A}_{0}, \beta$ : Velocity, amplitude and wavenumber of blowing and suction, respectively; $q, \bar{q}, \hat{q}$ : total flow field, mean flow, shape functions of disturbances; $u, v, p, T, w$ : streamwise velocity, wall-normal velocity, pressure, temperature, spanwise velocity; $\alpha, \omega$, : streamwise wavenumber of mode, angular frequency, dimensional frequency; $\mathcal{A}, \mathcal{B}, \mathcal{C}, \mathcal{L}, \mathcal{M}, \mathcal{H}$ : linear operators; V1,V2,V3,V4,V5,V6,V7: varicose modes; S1, S2: sinuous modes; $R 1, R 2, R 3, R 4, R 4$ : synchronization regions; $\phi, \phi_{a}$ : eigenfunction of the eigenvalue problem, of the adjoint eigenvalue problem; $S$ : an indicator of synchronization

\section{Acknowledgements}

The authors would like to thank Dr. Lei Zhao at Tianjin University, Dr. Ganglei Huang at Peking University and Dr. Guoliang Xu at China Aerodynamics Research and Development Center for helpful discussions.

\section{Authors' contributions}

All authors read and approved the final manuscript.

Funding

This work is funded by National Key Research and Development Project (2016YFA0401200).

\section{Availability of data and materials}

Parts of the data and materials are available upon request.

Competing interests

The authors declare that they have no competing interests. 


\section{References}

1. Hall P (1990) Görtler vortices in growing boundary layers: The leading edge receptivity problem, linear growth and the nonlinear breakdown stage. Mathematika 27:151-89

2. Floryan JM (1991) On the Görtler instability of boundary layers. Prog Aerop Sci 28:235-271

3. Saric WS (1994) Görtler vortices. Ann Rev Fluid Mech 26:379-409

4. Wu X, Zhao D, Luo J (2011) Excitation of steady and unsteady Görtler vortices by free-stream vortical disturbances. J Fluid Mech 682:66-100

5. Xu D, Zhang Y, Wu X (2017) Nonlinear evolution and secondary instability of steady and unsteady Görtler vortices induced by free-stream vortical disturbances. J Fluid Mech 829:681-730

6. Borodulin VI, Ivanov AV, Kachanov YS, Mischenko DA (2018) Systematic study of distributed excitation of unsteady Görtler modes by freestream vortices. Eur J Mech B-Fluids 68:167-183

7. Denier J, Hall P, Seddougui SO (1991) On the receptivity problem for Görtler vortices: vortex motions induced by wall roughness. Phil Trans $R$ Soc Lond A 335:51-85

8. Souza LF, Mendonca MT, Medeiros MAF, Kloker M (2004) Seeding of Görtler vortices through a suction and blowing strip. J Braz Soc Mech Sci Eng 26:269-279

9. Sescu A, Sassanis V, Haywood JS, Visbal M (2015) Study of the impact of localized roughness elements on Görtler instabilities. AIAA Paper:0275

10. Li F, Malik MR (1995) Fundamental and subharmonic secondary instabilities of Görtler vortices. J Fluid Mech 297:77-100

11. Ren J, Fu S (2015) Secondary instabilities of Görtler vortices in high-speed boundary layer flows. J Fluid Mech 781:388-421

12. Mitsudharmadi H, Winoto SH, Shah DA (2005) Splitting and merging of Görtler vortices. Phys Fluids 17:235-271

13. Mitsudharmadi H, Winoto SH, Shah DA (2006) Development of most amplified wavelength Görtler vortices. Phys Fluids 18:235-271

14. Winoto SH, Tandiono, Shah DA, Mitsudharmadi H (2008) Flows over concave surfaces: Development of pre-set wavelength Görtler vortices. Int J Fluid Mech Sys 1(1):10-23

15. Méndez M, Shadloo MS, Hadjadj A, Ducoin A (2018) Boundary layer transition over a concave surface caused by centrifugal instabilities. Comput Fluids 171:235-271

16. Chen X, Huang GL, Lee CB (2019) Hypersonic boundary layer transition on a concave wall: stationary görtler vortices. J Fluid Mech 865:1-40

17. Swearingen JD, Blackwelder RF (1987) The growth and breakdown of streamwise vortices in the presence of a wall. J Fluid Mech 182:255-290

18. Li F, Choudhari M, Chang C-L, Wu M, Greene P (2010) Development and breakdown of Görtler vortices in high speed boundary layers. AIAA Paper 2010-0705

19. Paredes P, Choudhari MM, Li F (2016) Transition due to streamwise streaks in a supersonic flat plate boundary layer. Phys Rev Fluids 1:235-271

20. Paredes P, Choudhari MM, Li F (2017) Instability wave-streak interactions in a supersonic boundary layer. J Fluid Mech 831:524-553

21. Chen X, Zhu Y, Lee C (2017) Interactions between second mode and low-frequency waves in a hypersonic boundary layer. J Fluid Mech 820:693-735

22. Li X, Fu D, Ma Y (2008) Direct numerical simulation of hypersonic boundary-layer transition over a blunt cone. AlAA J 46:2899-2913

23. Boyd JP (2000) Chebyshev and Fourier Spectral Methods (2nd Ed). DOVER Publications, Inc, New York

24. Fedorov AV, Khokhlov AP (2001) Prehistory of instability in a hypersonic boundary layer. Theoret Comput Fluid Dyn 14:359-375

25. Hein S, Theofilis V (2004) On instability characteristics of isolated vortices and models of trailing-vortex systems. Comput Fluids 33:741-753

26. Ma Y, Zhong X (2003) Receptivity of a supersonic boundary layer over a flat plate Part 1 wave structures and interactions. J Fluid Mech 488:31-78

27. Paredes P, Choudhari MM, Li F (2017) Stabilization of hypersonic boundary layers by linear and nonlinear optimal perturbations. AIAA Paper 2017-3634

28. Moyes AJ, Paredes P, Kocian TS, Reed HL (2017) Secondary instability analysis of crossflow on a hypersonic yawed straight circular cone. J Fluid Mech 812:370-397

\section{Publisher's Note}

Springer Nature remains neutral with regard to jurisdictional claims in published maps and institutional affiliations. 